\title{
Final state interaction and a light mass "exotic" resonance
}

\author{
Steven D. Bass ${ }^{[a]}$ and Eugenio Marco ${ }^{[b]}$ \\ a ECT*, Strada delle Tabarelle 286, I-38050 Villazzano, Trento, Italy \\ ${ }^{b}$ Physik Department, Technische Universität München, \\ D-85747 Garching, Germany
}

\begin{abstract}
We investigate the possibility that the light-mass exotic mesons with $J^{P C}=$ $1^{-+}$observed at BNL and CERN may be resonances in the $\eta \pi$ and $\eta^{\prime} \pi$ systems associated with the anomalous glue which generates the $\eta^{\prime}$ mass in QCD.
\end{abstract}




\section{Introduction}

Recent experiments at BNL [1, 2] and CERN [3] have revealed evidence for QCD "exotic" meson states with quantum numbers $J^{P C}=1^{-+}$. These mesons are particularly interesting because the quantum numbers $J^{P C}=1^{-+}$are inconsistent with a simple quark-antiquark bound state suggesting a possible "valence" gluonic component - for example through coupling to the operator $\left[\bar{q} \gamma_{\mu} q G^{\mu \nu}\right]$. Two such exotics, denoted $\pi_{1}$, have been observed through $\pi^{-} p \rightarrow \pi_{1} p$ at BNL: with masses $1400 \mathrm{MeV}$ (in decays to $\eta \pi$ ) [1] and $1600 \mathrm{MeV}$ (in decays to $\eta^{\prime} \pi$ and $\rho \pi$ ) [2]. The $\pi_{1}(1400)$ state has also been observed in $\bar{p} N$ processes by the Crystal Barrel Collaboration at CERN [3]. These states are considerably lighter than the predictions (about 1900 $\mathrm{MeV}$ ) of quenched lattice QCD [4] and QCD inspired models [5] for the lowest mass $q \bar{q} g$ state with quantum numbers $J^{P C}=1^{-+}$. While chiral corrections may bring the lattice predictions down by about $100 \mathrm{MeV}$ [6] these results suggest that, perhaps, the "exotic" states observed by the experimentalists might involve significant meson-meson bound state contributions. Furthermore, the decays to $\eta$ or $\eta^{\prime}$ mesons plus a pion may hint at a possible connection to axial $\mathrm{U}(1)$ dynamics.

In this paper we investigate the possibility that the observed exotics may be resonances in $\eta-\pi$ and $\eta^{\prime}-\pi$ scattering, possibly induced by the non-perturbative glue which generates the $\eta^{\prime}$ mass - the famous axial U(1) problem of QCD [7]. Unitarisation methods for chiral Lagrangians have shown that it is possible to dynamically generate resonances in the meson-baryon and meson-meson sectors [8, 9, 10, 11]. In particular, in the meson-meson sector resonances like the $\sigma, f_{0}, a_{0}$ which decay into two particles have been succesfully generated using coupled channels and the Bethe-Salpeter equation.

We investigate the $\eta \pi$ and $\eta^{\prime} \pi$ systems through the Bethe-Salpeter equation in a coupled channels approach with potentials derived from the leading order, $O\left(p^{2}\right)$, axial U(1) extended chiral Lagrangian. Before we begin our coupled channels discussion first consider the scenario that the observed processes involved production of a simple "hybrid" meson with valence $q \bar{q} g$ wavefunction which decays to $\eta \pi$ and $\eta^{\prime} \pi$ through flavour-singlet (OZI-violating) gluonic coupling of the "valence gluon" to the $\eta$ and $\eta^{\prime}$. In Born approximation (no final state interaction) one would predict

$$
r_{\pi_{1}}=\frac{\Gamma\left(\pi_{1} \rightarrow \eta^{\prime} \pi\right)}{\Gamma\left(\pi_{1} \rightarrow \eta \pi\right)}=\cot ^{2} \theta \sqrt{\frac{\left(M_{\pi_{1}}^{2}-\left(m_{\eta^{\prime}}+m_{\pi}\right)^{2}\right)\left(M_{\pi_{1}}^{2}-\left(m_{\eta^{\prime}}-m_{\pi}\right)^{2}\right)}{\left(M_{\pi_{1}}^{2}-\left(m_{\eta}+m_{\pi}\right)^{2}\right)\left(M_{\pi_{1}}^{2}-\left(m_{\eta}-m_{\pi}\right)^{2}\right)}}
$$

where $\theta$ is the $\eta-\eta^{\prime}$ mixing angle. Taking $\theta=-18$ degrees [12, 13] Eq.(1) evaluates to $r_{1400}=\Gamma\left(\pi_{1}(1400) \rightarrow \eta^{\prime} \pi\right) / \Gamma\left(\pi_{1}(1400) \rightarrow \eta \pi\right)=5.7$ and $r_{1600}=\Gamma\left(\pi_{1}(1600) \rightarrow\right.$ $\left.\eta^{\prime} \pi\right) / \Gamma\left(\pi_{1}(1600) \rightarrow \eta \pi\right)=6.8$ for the 1400 and $1600 \mathrm{MeV}$ states. The absence of an observed signal for $\pi_{1}(1400) \rightarrow \eta^{\prime} \pi$ and $\pi_{1}(1600) \rightarrow \eta \pi$ suggests that this scenario is too simple and that final state interaction and/or other hadronic physics is important to understanding the observed exotics.

We now discuss the chiral Lagrangian in Section 2 and use this in Section 3 within the Bethe-Salpeter formalism to investigate possible resonance structures with exotic quantum numbers. 


\section{The low-energy effective Lagrangian}

We start from the low-energy effective Lagrangian [14, 15]

$$
\begin{aligned}
\mathcal{L}_{\mathrm{m}}= & \frac{F_{\pi}^{2}}{4} \operatorname{Tr}\left(\partial^{\mu} U \partial_{\mu} U^{\dagger}\right)+\frac{F_{\pi}^{2}}{4} \operatorname{Tr} \chi_{0}\left(U+U^{\dagger}\right) \\
+ & \frac{1}{2} i Q \operatorname{Tr}\left[\log U-\log U^{\dagger}\right]+\frac{3}{\tilde{m}_{\eta_{0}}^{2} F_{0}^{2}} Q^{2} .
\end{aligned}
$$

where $U$ is the unitary meson matrix

$$
U=\exp \left(i \frac{\phi}{F_{\pi}}+i \sqrt{\frac{2}{3}} \frac{\eta_{0}}{F_{0}}\right)
$$

Here

$$
\phi=\sqrt{2}\left(\begin{array}{ccc}
\frac{1}{\sqrt{2}} \pi^{0}+\frac{1}{\sqrt{6}} \eta_{8} & \pi^{+} & K^{+} \\
\pi^{-} & -\frac{1}{\sqrt{2}} \pi^{0}+\frac{1}{\sqrt{6}} \eta_{8} & K^{0} \\
K^{-} & \bar{K}^{0} & -\frac{2}{\sqrt{6}} \eta_{8}
\end{array}\right)
$$

denotes the octet of would-be Goldstone bosons associated with spontaneous chiral $S U(3)_{L} \otimes S U(3)_{R}$ breaking, $\eta_{0}$ is the singlet boson and $Q$ denotes the topological charge density $Q=\frac{\alpha_{s}}{4 \pi} G_{\mu \nu} \tilde{G}^{\mu \nu}$. The pion decay constant $F_{\pi}=92.4 \mathrm{MeV} ; F_{0}$ renormalises the flavour-singlet decay constant and $\chi_{0}=\operatorname{diag}\left[m_{\pi}^{2}, m_{\pi}^{2},\left(2 m_{K}^{2}-m_{\pi}^{2}\right)\right]$ is the meson mass matrix.

The $U_{A}(1)$ gluonic potential involving the topological charge density is introduced to generate the gluonic contribution to the $\eta$ and $\eta^{\prime}$ masses and to reproduce the anomaly [16, 17] in the divergence of the gauge-invariantly renormalised flavoursinglet axial-vector current, viz.

$$
\partial^{\mu} J_{\mu 5}=\sum_{k=1}^{f} 2 i\left[m_{k} \bar{q}_{k} \gamma_{5} q_{k}\right]_{G I}+N_{f}\left[\frac{\alpha_{s}}{4 \pi} G_{\mu \nu} \tilde{G}^{\mu \nu}\right]_{G I}^{\mu^{2}}
$$

where

$$
J_{\mu 5}=\left[\bar{u} \gamma_{\mu} \gamma_{5} u+\bar{d} \gamma_{\mu} \gamma_{5} d+\bar{s} \gamma_{\mu} \gamma_{5} s\right]_{G I}^{\mu^{2}}
$$

The gluonic term $Q$ is treated as a background field with no kinetic term. It may

be eliminated through its equation of motion to generate a gluonic mass term for the singlet boson, viz.

$$
\frac{1}{2} i Q \operatorname{Tr}\left[\log U-\log U^{\dagger}\right]+\frac{3}{\tilde{m}_{\eta_{0}}^{2} F_{0}^{2}} Q^{2} \mapsto-\frac{1}{2} \tilde{m}_{\eta_{0}}^{2} \eta_{0}^{2}
$$

Coupling the Lagrangian (2) to the baryon octet allows one to study the $\eta$-nucleon and $\eta^{\prime}$-nucleon interactions [18, 19] where one finds a gluonic induced contact term 18 in the low-energy $p p \rightarrow p p \eta$ and $p p \rightarrow p p \eta^{\prime}$ reactions, which are presently under vigorous experimental study at CELSIUS [20] and COSY 21]. 
In this paper we shall be interested in meson scattering processes involving $\eta^{\prime} \pi \leftrightarrow$ $\left(\eta^{\prime} \pi, \eta \pi\right)$. Fourth-order terms in the meson fields are induced by the first two terms in Eq.(2) and also by the $U_{A}(1)$ invariant term [22]

$$
\mathcal{L}_{m 2 Q}=\lambda Q^{2} \operatorname{Tr} \partial_{\mu} U \partial^{\mu} U^{\dagger}
$$

which is important in describing the $\eta \eta^{\prime} \pi \pi$ system. After we eliminate $Q$ through its equation of motion the $Q$ dependent part of the effective Lagrangian $(2)+(8)$ becomes

$$
\begin{aligned}
\mathcal{L}_{Q} & =-\frac{1}{2} \tilde{m}_{\eta_{0}}^{2} \eta_{0}^{2}+\frac{1}{6} \lambda \tilde{m}_{\eta_{0}}^{4} \eta_{0}^{2} F_{0}^{2} \operatorname{Tr} \partial_{\mu} U \partial^{\mu} U^{\dagger}+\ldots \\
& =-\frac{1}{2} \tilde{m}_{\eta_{0}}^{2} \eta_{0}^{2}+\frac{3}{2} \eta_{0}^{2} \beta\left(\frac{1}{F_{\pi}}\right)^{2} \partial^{\mu} \pi_{a} \partial_{\mu} \pi_{a}+\frac{3}{2} \eta_{0}^{2} \beta\left(\frac{1}{F_{0}}\right)^{2} \partial_{\mu} \eta_{0} \partial^{\mu} \eta_{0}+\ldots
\end{aligned}
$$

where we have set $\lambda \equiv \frac{9}{2 F_{0}^{2} \tilde{m}_{\eta_{0}}^{4}} \beta$. In general, one can expect OZI violation wherever a coupling involving the $Q$-field occurs. The value of $F_{0}$ is usually determined from the decay rate for $\eta^{\prime} \rightarrow 2 \gamma$. In QCD one finds the relation [23]

$$
\frac{2 \alpha}{\pi}=\sqrt{\frac{3}{2}} F_{0}\left(g_{\eta^{\prime} \gamma \gamma}-g_{Q \gamma \gamma}\right)
$$

The observed decay rate [24] is consistent [12] with the OZI prediction for $g_{\eta^{\prime} \gamma \gamma}$ if $F_{0}$ and $g_{Q \gamma \gamma}$ take their OZI values: $F_{0} \simeq F_{\pi}$ and $g_{Q \gamma \gamma}=0$. In the rest of this paper we set $F_{0}=F_{\pi}$.

\section{$3 \quad$ Resonances in $\eta \pi$ and $\eta^{\prime} \pi$ scattering}

From the Lagrangian of Eqs. (21) and (8) one obtains the $T$ matrix elements at tree level, which, in this paper, we will call potentials $V$. We label the channels: $1=K \bar{K}, 2=\pi \eta_{8}, 3=\pi \eta_{0}$. The potentials involving the $\pi \eta_{8}$ and $K \bar{K}$ channels are derived from the $\mathrm{SU}(3)$ part of the Lagrangian and are given in Ref. [10. We write below the three additional combinations involving the singlet boson that we will need in our coupled channels analysis:

$$
\begin{gathered}
V_{13}=\frac{\sqrt{3}}{9 f_{\pi}^{2}}\left(2 m_{K}^{2}+m_{\pi}^{2}\right), \\
V_{23}=\frac{-\sqrt{2}}{3 f_{\pi}^{2}} m_{\pi}^{2},
\end{gathered}
$$

and

$$
V_{33}=\frac{-2}{3 f_{\pi}^{2}} m_{\pi}^{2}+\frac{3 \beta}{f_{\pi}^{2}}\left(t-2 m_{\pi}^{2}\right)
$$

We work in the one mixing angle scheme

$$
\begin{aligned}
|\eta\rangle & =\cos \theta\left|\eta_{8}\right\rangle-\sin \theta\left|\eta_{0}\right\rangle \\
\left|\eta^{\prime}\right\rangle & =\sin \theta\left|\eta_{8}\right\rangle+\cos \theta\left|\eta_{0}\right\rangle
\end{aligned}
$$


where the mixing angle $\theta$ is taken as -18 degrees [12, 13]. We refer the reader to 25, 26 for a discussion of the two-mixing angle scheme which enters if the chiral Lagrangian is extended to $O\left(p^{4}\right)$ in the meson momenta.

In order to generate the resonances we follow the method developed in Ref. [10], where the $a_{0}, \sigma$ and $f_{0}$ are generated dynamically using coupled channels and the Bethe-Salpeter equation. One can write the $T$ matrix as

$$
T_{i j}=V_{i j}+\sum_{k} V_{i k} G_{k} T_{k j}
$$

and then extract $T$, which in matrix form can be written as $T=(1-V G)^{-1} V$ where $G_{k}$ represents a loop with the mesons of channel $k$. The meson loop is regularised with a three-momentum cut-off which, following [10, 27], we take as $\Lambda=900 \mathrm{MeV}$ for all three channels. This three-momentum momentum cut-off is the only model parameter in our calculation and its value is fixed in order to reproduce the $a_{0}(980)$ mass and width. A change of $\pm 100 \mathrm{MeV}$ in the value of $\Lambda$ induces a change of \pm 30 $\mathrm{MeV}$ for the $a_{0}(980)$ pole mass and no variation of its width.

We investigate possible $s$ and $p$-wave resonances via the projection

$$
T_{L}=\frac{1}{2} \int_{-1}^{1} d \cos \theta P_{L}(\cos \theta) T
$$

For $s$-waves all three channels are involved, whereas for $p$-waves there is a decoupling of the channels, since they have different quantum numbers. $K \bar{K}$ has $J^{P C}=1^{--}$, whereas $\pi \eta$ and $\pi \eta^{\prime}$ are in $J^{P C}=1^{-+}$- the quantum numbers of the exotic. Here we consider only $p$ waves in the $J^{P C}=1^{-+}$channel.

The parameter $\beta$ can be determined via the $\eta^{\prime}$ decay into $\pi \pi \eta$,

$$
T_{\eta^{\prime} \rightarrow \eta \pi^{0} \pi^{0}}=\frac{1}{2} \sin 2 \theta\left(T_{\eta_{8} \rightarrow \eta_{8} \pi^{0} \pi^{0}}-T_{\eta_{0} \rightarrow \eta_{0} \pi^{0} \pi^{0}}\right)+\cos 2 \theta T_{\eta_{0} \rightarrow \eta_{8} \pi^{0} \pi^{0}}
$$

where $T_{\eta_{8} \rightarrow \eta_{8} \pi^{0} \pi^{0}}=\frac{-m_{\pi}^{2}}{3 f_{\pi}^{2}}$ 10, $T_{\eta_{0} \rightarrow \eta_{8} \pi^{0} \pi^{0}}=V_{23}$ and $T_{\eta_{0} \rightarrow \eta_{0} \pi^{0} \pi^{0}}=V_{33}$. One finds two possible solutions: $\beta=-0.625$ and $\beta=0.86$. We can investigate the resonance structures which result from each of these two possibilities and try to extract consequences.

With $\beta=-0.625$ a resonance structure appears in the $p$-wave amplitude. In Fig. 1 we show the amplitude squared for the different channels involved. One can observe a resonance structure at around $1400 \mathrm{MeV}$, most prominent in the $\pi \eta^{\prime} \rightarrow \pi \eta^{\prime}$ channel. By looking at the second Riemann sheet on the complex $s$ plane the pole can be found at $(1399,-153) \mathrm{MeV}$, a result remarkably close to the experimentally determined values for the $\pi_{1}(1400)$ : mass $1370 \pm 16 \mathrm{MeV}$ and width $385 \pm 40 \mathrm{MeV}$. With this value of $\beta$, there is no $s$-wave pole around $1450 \mathrm{MeV}$. Since our exotic is built exclusively through the $p$-wave part of the $\pi \eta_{0} \rightarrow \pi \eta_{0}$ amplitude, it decays mainly (86\%) into $\pi \eta^{\prime}$ and $14 \%$ to $\pi \eta$. These values clearly indicate that one would need to include higher order terms to get the branching ratios correct. However, these new terms would not change the position of the exotic, which is determined by the term $(1-V G)^{-1}$ with a $\pi \eta^{\prime}$ in the loop. The new terms involving higher order $\pi \eta_{8}$ contributions could act to increase the amplitude for $\pi \eta_{8} \rightarrow \pi \eta_{8}$ while keeping the same resonance structure. 
The opposite situation is found in the second scenario, taking $\beta=0.86$. Here we find an s-wave resonance structure around $1300 \mathrm{MeV}$, see Fig. 2, where all the different squared amplitudes are shown. Looking at the complex $s$ plane we find the pole at $(1286,-93) \mathrm{MeV}$. This resonance structure is a candidate for the scalar $a_{0}(1450)$ which has measured width $265 \pm 13 \mathrm{MeV}$ [24]. The branching ratios are: $39 \%$ into channel 1, $40 \%$ into channel 2 and $21 \%$ into channel 3 . No resonance structure appears in the $p$-wave amplitude with $\beta=0.86$.

These results are essentially insensitive to variations in the loop-momentum cutoff $\Lambda$. For the scalar $a_{0}(1450)$ candidate varying the cut-off parameter $\Lambda$ by \pm 100 $\mathrm{MeV}$ changes the mass of the scalar resonance by approximately $\pm 10 \mathrm{MeV}$. Decreasing $\Lambda$ to $800 \mathrm{MeV}$ increases the width by about $40 \mathrm{MeV}$ while increasing $\Lambda$ to 1 $\mathrm{GeV}$ decreases the width by just $2 \mathrm{MeV}$. For the resonance in the $p$-wave amplitude a change in $\Lambda$ by $\pm 100 \mathrm{MeV}$ induces no variation of the resonance mass, while its width changes by $\pm 100 \mathrm{MeV}$.

One can easily understand why different resonances are generated with the different signs of $\beta$. In order to have attraction the potential has to have a negative sign given our conventions. Once the $s$-wave is projected in $V_{33}$, there are two terms in it, a negative term which depends on the masses (see Eq. (13)) and the $\beta$ term with a minus sign in front of it. A positive $\beta$ adds attraction and the scalar resonance is generated. On the contrary a negative sign generates repulsion and the resonance is not generated. The $p$-wave part has the opposite behaviour. It has a $\beta$ term with a positive sign and thus only a negative value of $\beta$ generates the required attraction to generate the resonance.

Although we have no way of discarding any of the solutions and possibly higher order terms would be needed to have a more complete picture, it seems clear that this simple calculation illustrates the possibility to describe the appearance of exotics by means of a coupled channel treatment of the $\pi \eta$ and $\pi \eta^{\prime}$ systems. The topological charge density mediates the coupling of the light-mass exotic state to the $\eta \pi$ and $\eta^{\prime} \pi$ channels in our calculation.

\section{Acknowledgements}

We thank K. Seth and W. Weise for helpful discussions. This work was supported in part by the Alexander von Humboldt Foundation. 


\section{References}

[1] The E852 Collaboration (D.R. Thompson et al.) Phys. Rev. Lett. 79 (1997) 1630; (S.U. Chung et al.) Phys. Rev. D60 (1999) 092001.

[2] The E852 Collaboration (G.S. Adams et al.) Phys. Rev. Lett. 81 (1998) 5760; (E.I. Ivanov et al.) Phys. Rev. Lett. 86 (2001) 3977.

[3] The Crystal Barrel Collaboration (A. Abele et al.) Phys. Lett. B423 (1998) 246.

[4] P. Lacock et al. (UKQCD), Phys. Rev. D54 (1996) 6997;

P. Lacock et al. (UKQCD), Phys. Lett. B401 (1997) 308;

C. Bernard et al. (MILC) Phys. Rev. D56 (1997) 7039;

C. Bernard et al. (MILC) Nucl. Phys. B (Proc. Suppl.) 53 (1997) 228;

C. Michael, hep-ph/0101287.

[5] N. Isgur, R. Kokoski and J. Paton, Phys. Rev. Lett. 54 (1985) 869;

F.E. Close and P.R. Page, Nucl. Phys. B443 (1995) 233;

T. Barnes, F.E. Close and E.S. Swanson, Phys. Rev. D52 (1995) 5242.

[6] A.W. Thomas and A.P. Szczepaniak, hep-ph/0106080.

[7] G.M. Shore, Zuoz lecture, hep-ph/9812354.

[8] N. Kaiser, P.B. Siegel and W. Weise, Nucl. Phys. A594 (1995) 325; Phys. Lett. B362 (1995) 23.

[9] E. Oset and A. Ramos, Nucl. Phys. A635 (1998) 99.

[10] J.A. Oller and E. Oset, Nucl. Phys. A620 (1997) 438; (E) A652 (1997) 407.

[11] J. A. Oller, E. Oset and J. R. Pelaez, Phys. Rev. D59 (1999) 074001; (E) D60 (1999) 099906.

[12] F.J. Gilman and R. Kauffman, Phys. Rev. D36 (1987) 2761; (E) D37 (1988) 3348 .

[13] P. Ball, J.M. Frere and M. Tytgat, Phys. Lett. B365 (1996) 367.

[14] P. Di Vecchia and G. Veneziano, Nucl. Phys. B171 (1980) 253.

[15] C. Rosenzweig, J. Schechter and C.G. Trahern, Phys. Rev. D21 (1980) 3388;

P. Nath and R. Arnowitt, Phys. Rev. D23 (1981) 473.

[16] S.L. Adler, Phys. Rev. 177 (1969) 2426.

[17] J.S. Bell and R. Jackiw, Nuovo Cimento 60A (1969) 47.

[18] S. D. Bass, Phys. Lett B463 (1999) 286; hep-ph/0006348.

[19] S. D. Bass, S. Wetzel and W. Weise, Nucl. Phys. A686 (2001) 429. 
[20] The CELSIUS Collaboration (H. Calen et al.), Phys. Rev. Lett. 80 (1998) 2069; Phys. Rev. C58 (1998) 2667.

[21] The COSY-11 Collaboration (P. Moskal et al.), Phys. Rev. Lett. 80 (1998) 3202; Phys. Lett. B474 (2000) 416.

[22] P. Di Vecchia, F. Nicodemi, R. Pettorino and G. Veneziano, Nucl. Phys. B181 (1981) 318.

[23] G. M. Shore and G. Veneziano, Nucl. Phys. B381 (1992) 3;

G. M. Shore, Nucl. Phys. B569 (2000) 107.

[24] The Particle Data Group, C. Caso et al., Eur. Phys. J C3 (1998) 1.

[25] H. Leutwyler, Nucl. Phys. B (Proc. Suppl.) 64 (1998) 223;

R. Kaiser and H. Leutwyler, hep-ph/9806336.

[26] T. Feldmann, Int. J. Mod. Phys. A15 (2000) 159.

[27] E. Marco, S. Hirenzaki, E. Oset and H. Toki, Phys. Lett. B470 (1999) 20. 


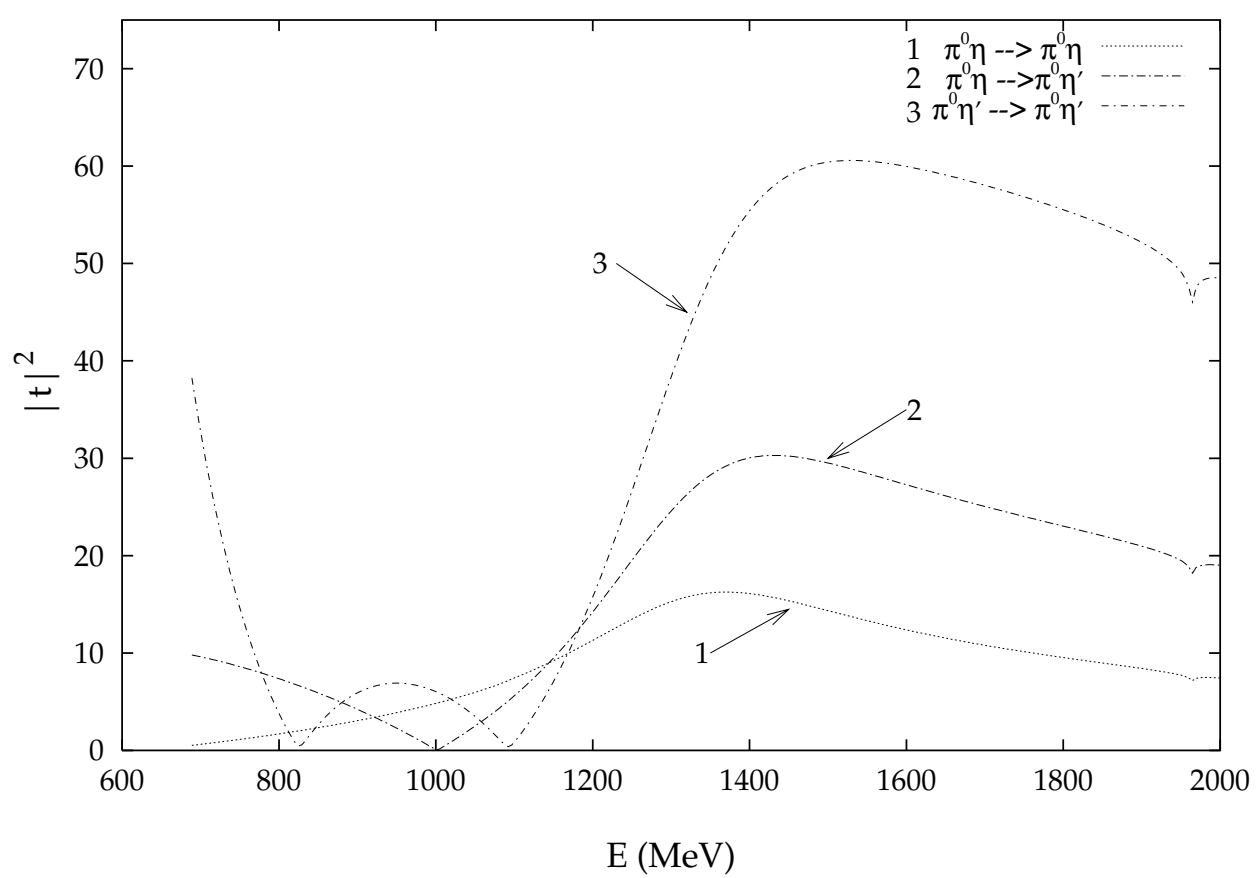

Figure 1:

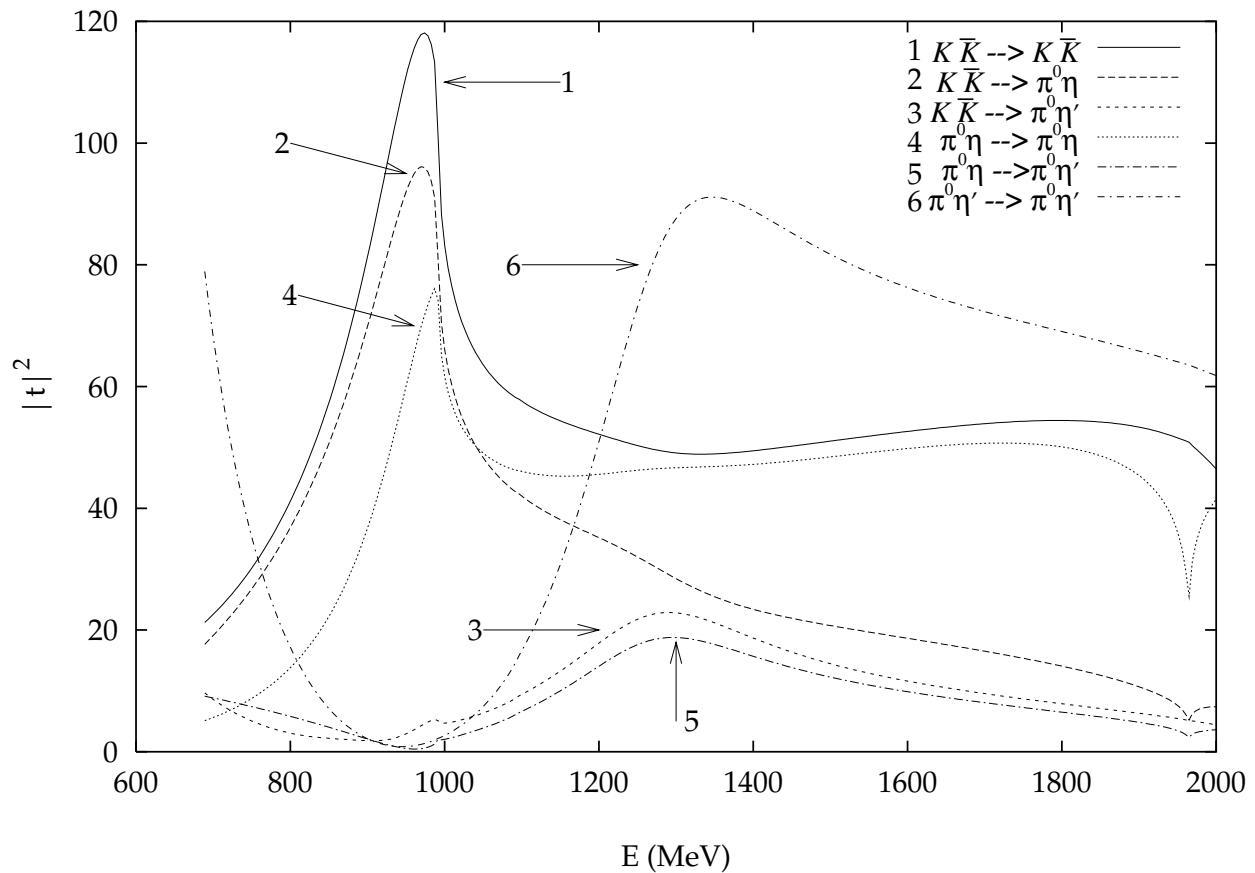

Figure 2: 